\title{
On a New Definition of the Fractional Difference
}

\author{
By Henry L. Gray and Nien fan Zhang
}

\begin{abstract}
A new definition of the fractional difference is introduced. Many properties based on this definition are established including an extensive exponential law and the important Leibniz rule. The results are then applied to solving second-order linear difference equations.
\end{abstract}

1. Introduction. Although the notions of fractional calculus date back to Euler, the idea of a fractional difference is more current. In one of the more extensive papers on the subject Diaz and Osler [1] defined the fractional difference by the rather natural approach of allowing the index of differencing, in the standard expression for the $n$th difference, to be any real or complex number, i.e.,

$$
\Delta^{\alpha} f(x)=\sum_{k=0}^{\infty}(-1)^{k}\left(\begin{array}{l}
\alpha \\
k
\end{array}\right) f(x+\alpha-k),
$$

where $\alpha$ is any real or complex number.

The definition furnished by (1.1) can be shown to result in an operator with many appealing properties. Unfortunately, however, it has no general exponential law, i.e., no law of the form $\Delta^{r+s} f(x)=\Delta^{r} \Delta^{s} f(x)$ without rather severe restrictions on $r$ and $s$. See Isaacs [4]. In addition, it has the shortcoming of requiring values of $f$ at nonintegral values of its argument. In many applied problems where one wishes to make use of the fractional difference, only values of $f$ at integral values of its argument are known. For example, in a discrete time series $f(x)=y_{x}$, $x=0, \pm 1, \pm 2, \ldots, \Delta^{\alpha}$ in (1.1) is not applicable. For this reason, the definition of Eq. (1.1) is not used in that field, but instead, Granger and Joyeux [2] and Hosking [3] have employed the following definition,

$$
\begin{aligned}
\nabla^{\alpha} f(x)=(1-B)^{\alpha} f(x) & =\sum_{k=0}^{\infty}(-1)^{k} \frac{\Gamma(\alpha+1)}{\Gamma(\alpha-k+1) \Gamma(k+1)} B^{k} f(x) \\
& =\sum_{k=0}^{\infty}(-1)^{k}\left(\begin{array}{l}
\alpha \\
k
\end{array}\right) f(x-k),
\end{aligned}
$$

where $\alpha$ is any real number and $B f(x)=f(x-1)$ is the standard backward shift operator. The notation $\nabla^{\alpha}$ is used since this definition is a natural extension of the backward difference operator. Making use of this latter definition, Granger and

Received March 14, 1986; revised November 24, 1986.

1980 Mathematics Subject Classification (1985 Revision). Primary 39A99; Secondary 39A10, 39A 70 .

Key words and phrases. Fractional difference, backward difference operator, Leibniz rule, difference equation. 
Joyeux and also Hosking have shown that the fractional difference can be used to study long memory time series.

In this paper we give a new definition of the fractional difference which also includes the notion of a fractional sum over a specified index set. One of the more important features of this new definition is that the sum corresponding to the one in (1.2) is finite. As a result, a general exponential law is obtained along with many other important properties. In addition, it is noted that the definition (1.2) is simply a limiting case of the definition given here. Finally, it is shown how these results can be employed to solve difference equations.

2. The Fractional Difference/Sum. For any complex number $\alpha$ and $\beta$ let $(\alpha)_{\beta}$ be defined as follows:

$$
(\alpha)_{\beta}= \begin{cases}\frac{\Gamma(\alpha+\beta)}{\Gamma(\alpha)} & \text { when } \alpha \text { and } \alpha+\beta \text { are neither zero nor negative } \\ 1 & \text { integers, } \\ 0 & \text { when } \alpha=\beta=0 \\ \text { undefined } & \text { when } \alpha=0, \beta \text { is not zero or a negative integer }\end{cases}
$$

In order to motivate our definition, consider the $n$-fold summation of $f$ from $a$ to $t$, i.e., let

$$
\stackrel{t}{S}^{n} f(t)=\sum_{k_{1}=a}^{t} \sum_{k_{2}=a}^{k_{1}} \ldots \sum_{k_{n}=a}^{k_{n-1}} f\left(k_{n}\right)
$$

where $t, k_{i}$ and $a$ are finite integers such that $a \leq k_{i} \leq k_{i-1} \leq t$. Then by repeated interchanging of summation it is easily shown that

$$
\stackrel{t}{S}^{n} f(t)=\frac{1}{\Gamma(n)} \sum_{k=a}^{t}(t-k+1)_{n-1} f(k) .
$$

The formula in (2.1) is in fact the analogue of Cauchy's formula for repeated integration. Moreover, the summation in (2.1) is well defined for $n=\alpha, \alpha$ any complex number not zero or a negative integer. The definition can be extended to zero and negative integers by noting that for $n$ a positive integer and $\alpha$ not zero or a negative integer, $\nabla f(t)=f(t)-f(t-1)$, and

$$
\begin{aligned}
& \frac{\nabla^{n}}{\Gamma(n+\alpha)} \sum_{k=a}^{t}(t-k+1)_{n+\alpha-1} f(k) \\
& \quad=\frac{1}{\Gamma(\alpha)} \sum_{k=a}^{t}(t-k+1)_{\alpha-1} f(k) .
\end{aligned}
$$

This leads us to the following definition of the fractional sum and difference.

Definition 1. For $\alpha$ any complex number, and $f$ defined over the integer set $\{a-n, a-n+1, \ldots, t\}$, the $\alpha$ th-order summation over $\{a, a+1, \ldots, t\}$ is defined 
by

$$
\stackrel{t}{S}^{\alpha} f(t)=\frac{\nabla^{n}}{\Gamma(n+\alpha)} \sum_{k=a}^{t}(t-k+1)_{n+\alpha-1} f(k),
$$

where $n=\max \left\{0, n_{0}\right\}, n_{0}$ an integer such that $0<\operatorname{Re}\left(\alpha+n_{0}\right) \leq 1$.

Definition 2. For $\alpha$ any complex number, the $\alpha$ th-order difference of $f(t)$ over $\{a, a+1, \ldots, t\}$ is defined by

$$
\stackrel{t}{a}^{\alpha} f(t)=\stackrel{t}{S}^{-\alpha} f(t)
$$

An immediate consequence of (2.4) is that if $\alpha=p$, a nonnegative integer, in (2.4) then $\alpha=-p$ in (2.3), hence by definition, $n=p+1$ and we have

$$
{\underset{a}{t}}^{p} f(t)=\nabla^{p+1} \sum_{k=a}^{t} f(k)=\nabla^{p} f(t) .
$$

Our definitions are therefore consistent with differencing in the usual sense and are well defined for any real or complex number $\alpha$.

Since our emphasis will be primarily on the fractional difference analogy, we shall primarily utilize the notation of (2.4). An alternative form of (2.3) that we will often find convenient to use is obtained by noting

$$
\sum_{k=a}^{t}(t-k+1)_{n+\alpha-1} f(k)=\sum_{k=0}^{t-a}(k+1)_{n+\alpha-1} f(t-k)
$$

so that

$$
\stackrel{a}{t}^{\alpha} f(t)=\frac{\nabla^{n}}{\Gamma(n-\alpha)} \sum_{k=0}^{t-a}(k+1)_{n-\alpha-1} f(t-k) .
$$

\section{Properties of Fractional Difference.}

PROPERTY 1. For any complex number $\alpha$ and nonnegative integer $p$ such that $p-\alpha$ is not zero or a negative integer,

$$
\stackrel{t}{\nabla}^{\alpha} f(t)=\frac{\nabla^{p}}{\Gamma(p-\alpha)} \sum_{k=a}^{t}(t-k+1)_{p-\alpha-1} f(k) .
$$

Proof. By definition (2.3) and (2.4),

$$
\stackrel{a}{a}^{\alpha} f(t)=\frac{\nabla^{n}}{\Gamma(n-\alpha)} \sum_{k=a}^{t}(t-k+1)_{n-\alpha-1} f(k),
$$

where $n=\max \left\{0, n_{0}\right\}, n_{0}$ an integer such that $0<\operatorname{Re}\left(n_{0}-\alpha\right) \leq 1$. 
Therefore, the result holds for $p=n$ trivially. Consider thus two cases:

(i) $p>n$. First let $p=n+1$. Then

$$
\begin{aligned}
& \frac{\nabla^{n+1}}{\Gamma(n+1-\alpha)} \sum_{k=a}^{t}(t-k+1)_{n+1-\alpha-1} f(k) \\
& =\frac{\nabla^{n}}{\Gamma(n+1-\alpha)}\left\{\nabla \sum_{k=a}^{t}(t-k+1)_{n-\alpha} f(k)\right\} \\
& =\frac{\nabla^{n}}{\Gamma(n+1-\alpha)}\{\Gamma(n-\alpha+1) f(t) \\
& \left.\quad+\sum_{k=a}^{t-1}\left[(t-k+1)_{n-\alpha}-(t-k)_{n-\alpha}\right] f(k)\right\} \\
& =\frac{\nabla^{n}}{\Gamma(n+1-\alpha)}\{\Gamma(n-\alpha+1) f(t) \\
& \left.\quad+(n-\alpha) \sum_{k=a}^{t-1}(t-k+1)_{n-\alpha-1} f(k)\right\} \\
& =\frac{\nabla^{n}}{\Gamma(n-\alpha)} \sum_{k=a}^{t}(t-k+1)_{n-\alpha-1} f(k) \\
& =\nabla_{a}^{\alpha} f(t) .
\end{aligned}
$$

Now suppose for some $p>n$

$$
\stackrel{t}{\nabla}^{\alpha} f(t)=\frac{\nabla^{p}}{\Gamma(p-\alpha)} \sum_{k=a}^{t}(t-k+1)_{p-\alpha-1} f(k) .
$$

Then, by using the same argument as before,

$$
\frac{\nabla^{p+1}}{\Gamma(p+1-\alpha)} \sum_{k=a}^{t}(t-k+1)_{p+1-\alpha-1} f(k)=\frac{\nabla^{p}}{\Gamma(p-\alpha)} \sum_{k=a}^{t}(t-k+1)_{p-\alpha-1} f(k) .
$$

Hence by induction, case (i) is proved.

(ii) $p<n$, i.e., $p=n-1, n-2, \ldots, 0$. By reversing the process of (3.2), case (ii) is easily shown. Then the proof is completed.

Property 1 and our observation in (2.5) can now be used to show that the definition in (1.2) is simply a limiting case of (2.4). That is, when $\alpha$ is not zero or a positive integer, by taking $p=0$ in Property 1 and noting that

$$
(-1)^{k} \frac{\Gamma(k-\alpha)}{\Gamma(-\alpha)}=\frac{\Gamma(\alpha+1)}{\Gamma(\alpha-k+1)}
$$

we have

$$
\begin{aligned}
\lim _{a \rightarrow-\infty} \stackrel{t}{\nabla}^{\alpha} f(t) & =\frac{1}{\Gamma(-\alpha)} \sum_{k=0}^{\infty}(k+1)_{-\alpha-1} f(t-k)=\sum_{k=0}^{\infty}(-1)^{k}\left(\begin{array}{l}
\alpha \\
k
\end{array}\right) f(t-k) \\
& =(1-B)^{\alpha} f(t)=\nabla^{\alpha} f(t)
\end{aligned}
$$


as defined in (1.2). From (2.5) the result also clearly holds when $\alpha=0$ or a positive integer. Henceforth, we will denote the above limit by replacing $a$ by $-\infty$, i.e., we define

$$
\stackrel{-\infty}{\nabla}_{-\infty}^{\alpha} f(t)=\lim _{a \rightarrow-\infty} \stackrel{t}{\nabla}_{a}^{\alpha} f(t)
$$

However, except where specifically indicated, " $a$ " will be assumed to be a finite integer.

PROPERTY 2. For $\alpha$ and $\beta$ any complex numbers

(i) if $\alpha$ and $\beta$ both are zero or positive integers, then

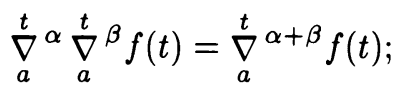

(ii) if $\alpha$ is any complex number and $\beta$ is not a positive integer, then

$$
\stackrel{\rightarrow}{\nabla}^{\alpha}{\underset{a}{t}}^{\beta} f(t)=\stackrel{i}{\nabla}^{\alpha+\beta} f(t)
$$

(iii) if $\alpha$ is not zero or a positive integer but $\beta$ is a positive integer, then

$$
\stackrel{t}{\nabla}_{a}^{\alpha} \stackrel{t}{\nabla}^{\beta} f(t)=\stackrel{t}{\nabla}^{\alpha+\beta} f(t)+\frac{1}{\Gamma(-\alpha)} \sum_{l=1}^{\beta} \sum_{j=a-l}^{a-1}(-1)^{l}\left(\begin{array}{c}
\beta \\
l
\end{array}\right)(t-l-j+1)_{-\alpha-1} f(j) .
$$

Proof. (i) is obvious.

(ii) Let $n_{1}, n_{2}$ be integers such that

$$
0<\operatorname{Re}\left(n_{1}-\alpha\right) \leq 1, \quad 0<\operatorname{Re}\left[n_{2}-(\alpha+\beta)\right] \leq 1,
$$

and let $n=\max \left\{0, n_{1}, n_{2}\right\}$. By Property 1 ,

$$
\begin{aligned}
\underset{a}{t}{ }_{a}^{\alpha} \nabla^{\beta} f(t) & =\frac{\nabla^{n}}{\Gamma(n-\alpha)} \sum_{k=a}^{t}(t-k+1)_{n-\alpha-1} \frac{1}{\Gamma(-\beta)} \sum_{j=a}^{k}(k-j+1)_{-\beta-1} f(j) \\
& =\frac{\nabla^{n}}{\Gamma(n-\alpha)} \frac{1}{\Gamma(-\beta)} \sum_{j=a}^{t} \sum_{k=j}^{t}(t-k+1)_{n-\alpha-1}(k-j+1)_{-\beta-1} f(j) .
\end{aligned}
$$

By shifting the index and algebraic manipulations, the above equals

$$
\nabla^{n} \sum_{j=a}^{t} \frac{f(j)}{\Gamma(t-j+1)} \sum_{k=0}^{t-j}\left(\begin{array}{c}
t-j \\
k
\end{array}\right)(n-\alpha)_{t-j-k}(-\beta)_{k}
$$

For $\alpha$ and $\beta$ complex numbers, when $\alpha, \beta$ and $\alpha+\beta$ are not zero or negative integers,

$$
(\alpha+\beta)_{n}=\sum_{k=0}^{n}\left(\begin{array}{l}
n \\
k
\end{array}\right)(\alpha)_{n-k} \beta_{k} \quad \text { for any positive integer } n .
$$

By (3.4), the expression (3.3) equals

$$
\begin{aligned}
& \nabla^{n} \sum_{j=a}^{t} \frac{f(j)}{\Gamma(t-j+1)}(n-\alpha-\beta)_{t-j} \\
& \quad=\frac{\nabla^{n}}{\Gamma(n-\alpha-\beta)} \sum_{j=a}^{t}(t-j+1)_{n-\alpha-\beta-1} f(j)=\stackrel{\nabla}{a}^{\alpha+\beta} f(t) .
\end{aligned}
$$


(iii) When $\alpha$ is not a zero or positive integer, but $\beta$ is a positive integer,

$$
\begin{aligned}
\underset{a}{t} \alpha \underset{a}{t}{ }^{\beta} f(t) & =\frac{1}{\Gamma(-\alpha)} \sum_{k=a}^{t}(t-k+1)_{-\alpha-1} \nabla^{\beta} f(k) \\
& =\frac{1}{\Gamma(-\alpha)} \sum_{k=a}^{t}(t-k+1)_{-\alpha-1} \sum_{l=0}^{\beta}\left(\begin{array}{c}
\beta \\
l
\end{array}\right)(-1)^{l} f(k-l) \\
& =\frac{1}{\Gamma(-\alpha)} \sum_{l=0}^{\beta} \sum_{k=a}^{t}(t-k+1)_{-\alpha-1}(-1)^{l}\left(\begin{array}{l}
\beta \\
l
\end{array}\right) f(k-l) \\
& =\frac{1}{\Gamma(-\alpha)} \sum_{l=0}^{\beta} \sum_{j=a-l}^{t-l}(t-l-j+1)_{-\alpha-1}(-1)^{l}\left(\begin{array}{c}
\beta \\
l
\end{array}\right) f(j) \\
& =\sum_{1}+\sum_{2},
\end{aligned}
$$

where

$$
\begin{gathered}
\sum_{1}=\frac{1}{\Gamma(-\alpha)} \sum_{l=0}^{\beta} \sum_{j=a}^{t-l}(t-l-j+1)_{-\alpha-1}(-1)^{l}\left(\begin{array}{c}
\beta \\
l
\end{array}\right) f(j), \\
\sum_{2}=\frac{1}{\Gamma(-\alpha)} \sum_{l=1}^{\beta} \sum_{j=a-l}^{a-1}(t-l-j+1)_{-\alpha-1}(-1)^{l}\left(\begin{array}{c}
\beta \\
l
\end{array}\right) f(j) .
\end{gathered}
$$

On the other hand, by applying Property 1 ,

$$
\begin{aligned}
\stackrel{a}{\nabla}^{\alpha+\beta} f(t) & =\frac{\nabla^{\beta}}{\Gamma(-\alpha)} \sum_{j=a}^{t}(t-j+1)_{-\alpha-1} f(j) \\
& =\frac{1}{\Gamma(-\alpha)} \sum_{l=0}^{\beta}(-1)^{l}\left(\begin{array}{c}
\beta \\
l
\end{array}\right) \sum_{j=a}^{t-l}(t-l-j+1)_{-\alpha-1} f(j)=\sum_{1} .
\end{aligned}
$$

Thus,

$$
\underset{a}{t} \underset{a}{\alpha}{\underset{a}{\beta}}^{\beta} f(t)=\nabla_{a}^{t}{ }^{\alpha+\beta} f(t)+\frac{1}{\Gamma(-\alpha)} \sum_{l=1}^{\beta} \sum_{j=a-l}^{a-1}(t-l-j+1)_{-\alpha-1}(-1)^{l}\left(\begin{array}{l}
\beta \\
l
\end{array}\right) f(j) .
$$

Note. In the special case $\alpha=-1, \beta=1$, the sum on the far right in (iii) just becomes $-f(a-1)$.

PROPERTY 3. When $\alpha$ is not a negative integer,

$$
\stackrel{\nabla}{a}^{\alpha} \stackrel{t}{a}^{-\alpha} f(t)=f(t)
$$

The proof is trivial by employing Property 2 .

PROPERTY 4. For c a constant,

$$
\stackrel{a}{\nabla}^{\alpha}[c f(t)+g(t)]=c{\underset{a}{t}}^{\alpha} f(t)+\stackrel{t}{a}^{\alpha} g(t) .
$$

The proof is trivial. 
PROPERTY 5 (LEIBNIZ RULE). If $m$ is a nonnegative integer,

$$
\nabla^{m} f(t) g(t)=\sum_{n=0}^{m}\left(\begin{array}{c}
m \\
n
\end{array}\right)\left[\nabla^{m-n} f(t-n)\right]\left[\nabla^{n} g(t)\right] \text {. }
$$

If $\alpha$ is not a nonnegative integer,

$$
\stackrel{a}{a}^{\alpha} f(t) g(t)=\sum_{n=0}^{t-a}\left(\begin{array}{l}
\alpha \\
n
\end{array}\right)\left[\begin{array}{c}
t-n \\
\nabla_{a}^{\alpha-n} f(t-n)
\end{array}\right]\left[\nabla^{n} g(t)\right] .
$$

Proof. If $m$ is a nonnegative integer, the result is well known. Suppose, therefore, that $\alpha$ is not zero or a positive integer. Then

$$
\stackrel{a}{a}^{\alpha} f(t) g(t)=\frac{1}{\Gamma(-\alpha)} \sum_{k=a}^{t}(t-k+1)_{-\alpha-1} f(k) g(k) .
$$

By induction it can be shown that

$$
\sum_{n=0}^{k}\left(\begin{array}{l}
k \\
n
\end{array}\right)(-1)^{n} \nabla^{n} g(t)=g(t-k)
$$

Thus,

$$
\stackrel{\operatorname{v}}{a}^{\alpha} f(t) g(t)
$$

can be written as

$$
\frac{1}{\Gamma(-\alpha)} \sum_{k=a}^{t}(t-k+1)_{-\alpha-1} f(k) \sum_{n=0}^{t-k}\left(\begin{array}{c}
t-k \\
n
\end{array}\right)(-1)^{n} \nabla^{n} g(t) .
$$

Since $\Gamma(\alpha+1) \Gamma(-\alpha) / \Gamma(\alpha-n+1) \Gamma(n-\alpha)=(-1)^{n}$ for any nonnegative integer $n$, the above expression becomes

$$
\begin{aligned}
\frac{1}{\Gamma(-\alpha)} & \sum_{k=a}^{t}(t-k+1)_{-\alpha-1} f(k) \sum_{n=0}^{t-k}\left(\begin{array}{c}
t-k \\
n
\end{array}\right) \frac{\Gamma(\alpha+1) \Gamma(-\alpha)}{\Gamma(\alpha-n+1) \Gamma(n-\alpha)} \nabla^{n} g(t) \\
= & \frac{1}{\Gamma(-\alpha)} \sum_{n=0}^{t-a} \sum_{k=a}^{t-n}(t-k+1)_{-\alpha-1} f(k)\left(\begin{array}{c}
t-k \\
n
\end{array}\right) \frac{\Gamma(\alpha+1) \Gamma(-\alpha)}{\Gamma(\alpha-n+1) \Gamma(n-\alpha)} \nabla^{n} g(t) \\
= & \sum_{n=0}^{t-a} \frac{\Gamma(\alpha+1)}{\Gamma(n+1) \Gamma(\alpha-n+1)} \\
& \times\left\{\frac{1}{\Gamma(n-\alpha)} \sum_{k=a}^{t-n}(t-n-k+1)_{n-\alpha-1} f(k)\right\} \nabla^{n} g(t) \\
= & \sum_{n=0}^{t-a}\left(\begin{array}{l}
\alpha \\
n
\end{array}\right) \nabla_{a}^{t-n} \alpha-n f(t-n) \nabla^{n} g(t) . \quad
\end{aligned}
$$

PROPERTY 6. If $p+1$ is not zero or a negative integer, then

(i) when $p+1-\alpha$ is not zero or a negative integer,

$$
\underset{a+1}{t} \alpha(t-a)_{p}=\frac{(t-a)_{p-\alpha}}{(p+1)_{-\alpha}}
$$

(ii) when $p+1-\alpha$ is zero or a negative integer,

$$
\stackrel{t}{a+1}^{\alpha}(t-a)_{p}=0 \text {. }
$$


Proof. When $\alpha$ is a positive integer, the result is obvious. Hence, we only consider the case that $\alpha$ is not zero or a positive integer.

(i) We have

$$
\begin{aligned}
\stackrel{t}{a+1}_{a}^{\alpha}(t-a)_{p} & =\frac{1}{\Gamma(-\alpha)} \sum_{j=a+1}^{t}(t-j+1)_{-\alpha-1}(j-a)_{p} \\
& =\frac{1}{\Gamma(-\alpha)} \sum_{j=a+1}^{t} \frac{\Gamma(t-j-\alpha)}{\Gamma(t-j+1)} \frac{\Gamma(j-a+p)}{\Gamma(j-a)} \\
& =\frac{1}{\Gamma(-\alpha)} \sum_{j=0}^{t-a-1} \frac{\Gamma(t-j-a-\alpha-1)}{\Gamma(t-j-a)} \frac{\Gamma(j+p+1)}{\Gamma(j+1)} \\
& =\frac{\Gamma(p+1)}{\Gamma(t-a)} \sum_{j=0}^{t-a-1}\left(\begin{array}{c}
t-a-1 \\
j
\end{array}\right)(-\alpha)_{t-a-1-j}(p+1)_{j} .
\end{aligned}
$$

Using (3.4) again,

$$
\stackrel{t}{\nabla}_{a+1}^{\alpha}(t-a)_{p}=\frac{\Gamma(p+1)}{\Gamma(t-a)}(p-\alpha+1)_{t-a-1}=\frac{(t-a)_{p-\alpha}}{(p+1)_{-\alpha}}
$$

(ii) When $p+1-\alpha$ is zero or a negative integer, say $p+1-\alpha=-m$, where $m$ is a positive integer or zero, then by Property 2 and (i),

$$
\begin{aligned}
& \underset{a+1}{t} \alpha(t-a)_{p}=\stackrel{t}{a+1}^{m+1} \stackrel{t}{\nabla+1}^{\alpha-m-1}(t-a)_{p} \\
& =\stackrel{\nabla}{a+1}^{m+1} \Gamma(p+1)=0 .
\end{aligned}
$$

Example 1.

$$
\stackrel{x}{\nabla}_{1}^{1 / 2}(x)_{1 / 2}=\frac{(x)_{0}}{(3 / 2)_{-1 / 2}}=\Gamma(3 / 2), \quad \stackrel{x}{\nabla}^{3 / 2}(x)_{1 / 2}=0 .
$$

PROPERTY 7. Let $B(\alpha, \beta)$ denote the Beta function when $\alpha, \beta$ are not zero or negative integers. For any real or complex numbers $\alpha$ and $p$ with $p+1, p+1-\alpha$ not zero or negative integers,

$$
\stackrel{t}{\nabla^{\alpha}} \frac{1}{B(t-a, p)}=\frac{p}{p-\alpha} \frac{1}{B(t-a, p-\alpha)}
$$

Proof. Follows at once from Property 6.

PROPERTY 8. For any $b$ with $|b|>1$,

(i) when $\alpha$ is zero or a positive integer,

$$
\nabla^{\alpha} b^{t}=\left(\frac{b-1}{b}\right)^{\alpha} b^{t}
$$

(ii) when $\alpha$ is not zero or a positive integer,

$$
\stackrel{a}{a}^{\alpha} b^{t}=\frac{b^{t}}{\Gamma(-\alpha)} \sum_{k=0}^{t-a} \frac{(k+1)_{-\alpha-1}}{b^{k}}
$$

(iii) when $a \rightarrow-\infty$, then for any complex number $\alpha$,

$$
\stackrel{\nabla}{-\infty}^{\alpha} b^{t}=\left(\frac{b-1}{b}\right)^{\alpha} b^{t}
$$


Proof. (i) Since $\nabla b^{t}=(b-1) b^{t} / b$, the result is obvious.

(ii) By using Property 1,

$$
\begin{aligned}
\underset{a}{t} \alpha b^{t} & =\frac{1}{\Gamma(-\alpha)} \sum_{k=0}^{t-a}(k+1)_{-\alpha-1} b^{t-k} \\
& =\frac{b^{t}}{\Gamma(-\alpha)} \sum_{k=0}^{t-a}(k+1)_{-\alpha-1} b^{-k} .
\end{aligned}
$$

(iii) From (i), the result clearly holds if $\alpha$ is zero or a positive integer. Therefore, assume $\alpha \neq 0,1,2, \ldots$ Then from (ii),

$$
\begin{aligned}
\underset{-\infty}{\nabla^{\alpha} b^{t}} & =\lim _{a \rightarrow-\infty} \frac{b^{t}}{\Gamma(-\alpha)} \sum_{k=0}^{t-a}(k+1)_{-\alpha-1} b^{-k} \\
& =\frac{b^{t}}{\Gamma(-\alpha)} \sum_{k=0}^{\infty}(k+1)_{-\alpha-1} b^{-k}=\frac{b^{t}}{\Gamma(-\alpha)} \sum_{k=0}^{\infty} \frac{\Gamma(k-\alpha)}{\Gamma(k+1)} \frac{1}{b^{k}} \\
& =\frac{b^{t}}{\Gamma(-\alpha)} \sum_{k=0}^{\infty} \int_{0}^{\infty} \frac{e^{-x} x^{k-\alpha-1}}{b^{k}} \frac{1}{k !} d x .
\end{aligned}
$$

By applying Fubini's theorem, it is easy to show that summation and integration can be interchanged. Thus,

$$
\begin{aligned}
\stackrel{-}{-\infty}^{\alpha} b^{t} & =\frac{b^{t}}{\Gamma(-\alpha)} \int_{0}^{\infty}\left[\sum_{k=0}^{\infty}\left(\frac{x}{b}\right)^{k} \frac{1}{k !}\right] x^{-\alpha-1} e^{-x} d x \\
& =\frac{b^{t}}{\Gamma(-\alpha)} \int_{0}^{\infty} e^{-x+x / b} x^{-\alpha-1} d x \\
& =\frac{b^{t}}{\Gamma(-\alpha)}\left(\frac{b}{b-1}\right)^{-\alpha} \Gamma(-\alpha)=\left(\frac{b-1}{b}\right)^{\alpha} b^{t}
\end{aligned}
$$

Example 2. When $b=2$,

$$
\stackrel{x}{\nabla}_{-\infty}^{\alpha} 2^{x}=2^{x-\alpha}
$$

Example 3. Let $f(t)$ be defined by

$$
f(t)= \begin{cases}1, & t=0, \\ 0, & t=1,2, \ldots\end{cases}
$$

Suppose $\alpha \neq 0,1,2, \ldots$; then by Property 1 ,

$$
\begin{aligned}
\underset{0}{\nabla^{\alpha}} f(t) & =\frac{1}{\Gamma(-\alpha)} \sum_{k=0}^{t}(t-k+1)_{-\alpha-1} f(t) \\
& =\frac{1}{\Gamma(-\alpha)}(t+1)_{-\alpha-1}=\frac{\Gamma(t-\alpha)}{\Gamma(-\alpha) \Gamma(t+1)}
\end{aligned}
$$

Note, if $\alpha=1,2, \ldots$, i.e., $\alpha$ is a positive integer,

$$
\begin{aligned}
\stackrel{\nabla}{\nabla}^{n} f(t) & =\nabla^{n} f(t)=\sum_{k=0}^{n}(-1)^{k}\left(\begin{array}{l}
n \\
k
\end{array}\right) f(t-k) \\
& =(-1)^{t}\left(\begin{array}{l}
n \\
t
\end{array}\right)
\end{aligned}
$$


which is the same result as obtained when $\alpha$ was not an integer, since

$$
(-1)^{t}=\frac{\Gamma(t-n)}{\Gamma(-n)} \cdot \frac{\Gamma(n-t+1)}{\Gamma(n+1)}
$$

for any positive integer $t$. It is interesting to note the index Property 2 in this example. That is, by proceeding directly for $\alpha$ and $\beta \neq 0$, or positive integers, we have

$$
\begin{aligned}
\stackrel{0}{\nabla}_{0}^{\alpha}{ }_{0}^{t}{ }^{\beta} f(t) & ={ }_{0}^{t} \alpha \frac{1}{\Gamma(-\beta)}(t+1)_{-\beta-1} \\
& =\frac{1}{\Gamma(-\beta)} \frac{(t+1)_{-\alpha-\beta-1}}{(-\beta)_{-\alpha}}
\end{aligned}
$$

by Property 6 . Therefore,

$$
\stackrel{t}{\nabla}_{0}^{\alpha}{\underset{0}{t}}^{\beta} f(t)=\frac{1}{\Gamma(-\alpha-\beta)}(t+1)_{-\alpha-\beta-1}=\stackrel{t}{\nabla}^{\alpha+\beta} f(t) .
$$

4. Applications. In this section we show how the fractional difference can be employed to determine the solution of a rather general class of second-order linear difference equations. Other such equations could be solved by the same approach. To demonstrate the ideas involved, we first consider a rather trivial example and then extend the technique to a more general situation.

Example 4. The Leibniz rule can be used to write standard difference equations in a "factored" form. For example, consider

$$
\stackrel{\nabla}{t}^{\alpha} t y(t)
$$

and apply the Leibniz rule to obtain

$$
\stackrel{t}{\nabla}_{0}^{\alpha} t y(t)=t \stackrel{t}{\nabla}_{0}^{\alpha} y(t)+\alpha \stackrel{t-1}{\nabla}_{0}^{\alpha-1} y(t-1)
$$

Then suppose $\alpha$ is not zero or a negative integer and let

$$
y(t)=\stackrel{t}{\nabla}^{-\alpha+1} Z(t)
$$

Then, using Property 2(ii),

$$
\begin{aligned}
\stackrel{t}{\nabla}^{\alpha} t y(t) & =t \stackrel{t}{\nabla}_{0}^{\alpha} \stackrel{t}{\nabla}^{-\alpha+1} Z(t)+\alpha Z(t-1) \\
& =t \nabla Z(t)+\alpha Z(t-1)=t Z(t)+(\alpha-t) Z(t-1)
\end{aligned}
$$

Thus the difference equation

$$
t Z(t)+(\alpha-t) Z(t-1)=0
$$

can be written in the "factored" form

$$
\stackrel{t}{\nabla}_{0}^{\alpha} t{\underset{0}{\nabla}}^{-\alpha+1} Z(t)=0
$$

This type of observation will be used in this section to demonstrate how fractional differences can be used to solve difference equations. 
THEOREM 4.1. Let $y(x)$ satisfy the difference equation

$$
(a x+b) y(x)+(c x+d) y(x-1)+(e x+f) y(x-2)=H(x)
$$

for all $x$ in some set $S$. Let $\mu$ satisfy the equation

$$
e \mu^{2}+c \mu+a=0
$$

and define

$$
\begin{aligned}
& \alpha=a-e \mu^{2} \\
& \beta=\frac{a b-a f \mu^{2}-2 e b \mu^{2}-e d \mu^{3}}{a-e \mu^{2}} \\
& \gamma=e \mu^{2} \\
& \delta=\frac{a f \mu^{2}+e b \mu^{2}+e d \mu^{3}}{a-e \mu^{2}} \\
& \nu=\frac{b+d \mu+f \mu^{2}}{a-e \mu^{2}} \\
& p_{1}(x)=\alpha x+\beta \\
& p_{2}(x)=\gamma x+\delta \\
& q(x)=\frac{p_{2}(x)}{p_{1}(x)+p_{2}(x)}
\end{aligned}
$$

Let $x_{0} \in S$ be such that $Q(x) \neq 0$ for $x \geq x_{0}$, where

$$
Q(x)=\prod_{i=x_{0}}^{x} q(i) .
$$

Then for all $x \in S$ such that $x>x_{0}$ the difference equation in (4.1) has the equivalent form

$$
\underset{x_{0}}{\nabla^{\nu}}\left[p_{1}(x)+p_{2}(x)\right] Q(x) \nabla Q^{-1}(x) \underset{x_{0}}{x^{-\nu+1}} \mu^{x} y(x)=\mu^{-x} H(x),
$$

provided $\mu>0$ and $\nu$ is not zero or a negative integer. We can assume $a-e \mu^{2} \neq$ 0 , since otherwise it is easy to show that Eq. (4.1) reduces to one with constant coefficients.

Proof. For any function $\phi(x)$, letting $\nabla=1-B$,

$$
\begin{aligned}
{\left[p_{2}(x)\right.} & \left.\nabla+p_{1}(x)\right] \phi(x) \\
& =\left\{\left[p_{2}(x)+p_{1}(x)\right]-p_{2}(x) B\right\} \phi(x) \\
& =\left[p_{1}(x)+p_{2}(x)\right][\phi(x)-q(x) \phi(x-1)] \\
& =\left[p_{1}(x)+p_{2}(x)\right] Q(x)\left[\frac{\phi(x)}{Q(x)}-\frac{\phi(x-1)}{Q(x-1)}\right] \\
& =\left[p_{1}(x)+p_{2}(x)\right] Q(x) \nabla Q^{-1}(x) \phi(x) .
\end{aligned}
$$

Thus,

$$
\begin{aligned}
& {\stackrel{x}{x_{0}}}^{x}\left[p_{2}(x)+p_{1}(x)\right] Q(x) \nabla Q^{-1}(x) \underset{x_{0}}{\stackrel{x}{\nabla}}-\nu+1 \mu^{x} y(x) \\
& ={\stackrel{x}{x_{0}}}^{x}\left[p_{2}(x) \nabla+p_{1}(x)\right]{\stackrel{x}{x_{0}}}^{-\nu+1} \mu^{x} y(x) \\
& =\underset{x_{0}}{\stackrel{x}{\nabla}} p_{2}(x) \underset{x_{0}}{\stackrel{x}{\nabla}}-\nu+2 \mu^{x} y(x)+\underset{x_{0}}{\stackrel{x}{\nabla}} p_{1}(x) \underset{x_{0}}{\stackrel{x}{\nabla}-\nu+1} \mu^{x} y(x) .
\end{aligned}
$$


But by the Leibniz rule, if we set

$$
g(x)=p_{2}(x) \text { and } \quad f(x)={\underset{x}{x_{0}}}^{x}-\nu+2 \mu^{x} y(x),
$$

we have

$$
\begin{aligned}
& \underset{x_{0}}{\nabla^{x}} p_{2}(x) \underset{x_{0}}{\stackrel{x}{\nabla}}-\nu+2 \mu^{x} y(x) \\
& \quad=p_{2}(x) \underset{x_{0}}{\stackrel{x}{\nabla}} \stackrel{x_{x_{0}}^{x}}{-\nu+2} \mu^{x} y(x)+\nu \nabla p_{2}(x) \underset{x_{0}}{\stackrel{x-1}{\nabla} \nu-1} \underset{x_{0}}{\stackrel{x-1}{\nabla}-\nu+2} \mu^{x-1} y(x-1)
\end{aligned}
$$

Similarly,

$$
\begin{aligned}
& \underset{x_{0}}{\stackrel{x}{\nabla}} p_{1}(x) \underset{x_{0}}{\stackrel{x}{\nabla}}-\nu+1 \mu^{x} y(x) \\
& \quad=p_{1}(x) \underset{x_{0}}{\stackrel{x}{\nabla}} \stackrel{x}{x_{0}}-\nu+1 \mu^{x} y(x)+\nu \alpha \underset{x_{0}}{\nabla-1} \nu \underset{x_{0}}{\stackrel{x-1}{\nabla}-\nu+1} \mu^{x-1} y(x-1) .
\end{aligned}
$$

Substituting (4.6) and (4.7) in (4.5) and collecting terms gives

$$
\begin{aligned}
\mu^{x}\left\{\left[p_{1}(x)+p_{2}(x)\right] y(x)+\left[\frac{-2}{\mu} p_{2}(x)+\frac{\nu}{\mu} \nabla p_{2}(x)-\frac{p_{1}(x)}{\mu}+\frac{\alpha \nu}{\mu}\right] y(x-1)\right. & \\
& \left.+\left[\frac{p_{2}(x)}{\mu^{2}}-\frac{\nu \nabla p_{2}(x)}{\mu^{2}}\right] y(x-2)\right\}
\end{aligned}
$$

or

$$
\begin{aligned}
& \mu^{x}\{[(\alpha+\gamma) x+(\beta+\delta)] y(x) \\
& +\left[-\frac{(\alpha+2 \gamma)}{\mu} x+\frac{\nu(\alpha+\gamma)-\beta-2 \delta}{\mu}\right] y(x-1) \\
& \left.+\left[\frac{\gamma}{\mu^{2}} x+\frac{\delta-\nu \gamma}{\mu^{2}}\right] y(x-2)\right\}
\end{aligned}
$$

Substituting for $\alpha, \beta, \gamma$ and $\delta$ in terms of $a, b, c, d, e$ and $f$ immediately yields (4.1). Consequently, by (4.8), when $\alpha, \beta, \gamma, \delta, \nu$ are determined by the system (4.2) and when $\mu>0$, the difference equation (4.1) has the equivalent form (4.4), since the process can be reversed.

By using Property 6(ii), we have the following lemma.

LEMMA 4.1. If $\alpha$ is not zero or a negative integer, then

$$
f(x)=\sum_{i=1}^{n} d_{i}\left(x-x_{0}+1\right)_{\alpha-i}
$$

is a solution of the difference equation

$$
{\stackrel{x}{x_{0}}}^{x} f(x)=0
$$

where $n$ is any finite positive integer and the $d_{i}$ 's are constants.

The equivalent factored form can now be used to obtain a solution of the equation by simply operating on both sides of the equation by the corresponding inverses. That is, applying Theorem 4.1 and Lemma 4.1, we have the following corollary. 
COROLlaRY 4.1. For the equation

$$
(a x+b) y(x)+(c x+d) y(x-1)+(e x+f) y(x-2)=0
$$

(i) when $\mu>0$ and $\nu$ is not an integer,

$$
\begin{aligned}
y(x)= & \mu^{-x} \underset{x_{0}}{\stackrel{x}{\nabla}}{ }^{\nu-1} Q(x) \underset{x_{0}}{\stackrel{x}{\nabla}}{ }^{-1} Q^{-1}(x)\left[p_{1}(x)+p_{2}(x)\right]^{-1} \sum_{j=1}^{n} d_{j}\left(x-x_{0}+1\right)_{\nu-j} \\
& +k \mu^{-x} \underset{x_{0}}{\stackrel{x}{\nabla}}{ }^{\nu-1} Q(x)
\end{aligned}
$$

is a solution of (4.9) for $x>x_{0}$, where $n$ is any finite positive integer, $k$ is an arbitrary constant, the $d_{j}$ 's are constants, and $x_{0}$ is finite;

(ii) when $\mu>0$ and $\nu \neq 0,-1,-2, \ldots$,

$$
y(x)=k \mu^{-x} \nabla^{\nu-1} Q(x)
$$

is a solution of (4.9), where $k$ is an arbitrary constant;

(iii) when $\mu>0$ and $\nu=0$,

$$
y(x)=k \mu^{-x}
$$

is a solution of (4.9), where $k$ is an arbitrary constant.

Although it has not been shown, we conjecture that for proper choice of the $d_{j}$ and $x_{0}$ the solution in (i) can be shown to be the general solution. Of course, one can use any of the solutions in (i) to find another linearly independent solution by standard methods and produce the general solution to (4.9).

COROLlARY 4.2. When $\mu>0$ and $\nu$ is not an integer, Eq. (4.1) has a particular solution

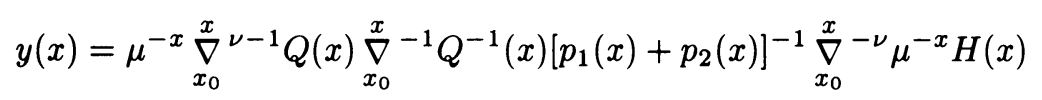

for $x>x_{0}$.

Example 5. Consider the following equation

$$
(3 x+4) y(x)-8(x+1) y(x-1)+4(x+2) y(x-2)=0 .
$$

By solving the system of equations (4.2),

$$
\alpha=2, \quad \beta=1, \quad \gamma=1, \quad \delta=3, \quad \mu=1 / 2, \quad \nu=1,
$$

and $p_{1}(x)=2 x+1, p_{2}(x)=x+3$. Then,

$$
q(x)=\frac{x+3}{3 x+4} \text { and } Q(x)=\prod_{i=-2}^{x} q(i),
$$

so that $x_{0}=-2$. The equivalent equation is thus

$$
{\underset{-2}{\nabla}}^{1}\left[p_{1}(x)+p_{2}(x)\right] Q(x) \nabla Q^{-1}(x)\left(\frac{1}{2}\right)^{x} y(x)=0 .
$$

By Corollary 4.1(ii) it is easy to see that $y(x)=k 2^{x} Q(x)$ is a solution, where $k$ is an arbitrary constant for $x>-2$.

A second solution can be obtained by using this solution to reduce the order of the equation and proceeding by standard methods or by (4.10). 
5. The Limiting Case. In several instances in the previous sections, we referred to the limiting case, i.e., the case $a=-\infty$. In this final section we explore that case more closely and give sufficient conditions for many of the previous properties to hold.

PROPERTY 1'. For any $\alpha$ and nonnegative integer $p$ such that $p-\alpha$ is not zero or a negative integer, when $\stackrel{-}{-\infty}^{\alpha} f(t)$ exists, then

$$
\stackrel{\nabla}{-\infty}^{\alpha} f(t)=\lim _{a \rightarrow-\infty} \frac{\nabla^{p}}{\Gamma(p-\alpha)} \sum_{k=a}^{t}(t-k+1)_{p-\alpha-1} f(k) .
$$

Proof. By the definition, let $n=\max \left\{0, n_{0}\right\}$ such that $0<\operatorname{Re}\left(n_{0}-\alpha\right) \leq 1$. Then

$$
\begin{aligned}
\underset{-\infty}{\nabla^{\alpha}} f(t) & =\lim _{a \rightarrow-\infty} \frac{\nabla^{n}}{\Gamma(n-\alpha)} \sum_{k=a}^{t}(t-k+1)_{n-\alpha-1} f(k) \\
& =\lim _{a \rightarrow-\infty} \frac{\nabla^{p}}{\Gamma(p-\alpha)} \sum_{k=a}^{t}(t-k+1)_{p-\alpha-1} f(k),
\end{aligned}
$$

since the two quantities under the limit are equal by Property 1 .

PROPERTY $2^{\prime}$. (i) If $\alpha$ and $\beta$ are both zero or positive integers, or

(ii) if $\alpha$ is any complex number and $\beta$ is not zero or a positive integer, and if
(a) $\stackrel{t}{\nabla_{-\infty}^{\beta}} f(t)$ exists;
(b) $\lim _{\substack{a \rightarrow-\infty \\ b \rightarrow-\infty}} \stackrel{t}{a}^{\alpha} \stackrel{t}{\nabla}_{b}^{\beta} f(t) \quad$ exists;
(c) $\stackrel{t}{\nabla}_{-\infty}^{\alpha+\beta-n} f(t)$ exists, where $n$ is defined as in the proof of Property 2, or

(iii) if $\alpha$ is not zero or a positive integer but $\beta$ is a positive integer, and
(a) $\underset{-\infty}{\nabla^{\alpha}} f(t) \quad$ exists;
(b) $\underset{-\infty}{\stackrel{t}{\nabla}} \stackrel{t}{\nabla} \beta f(t) \quad$ exists;

then

$$
{\underset{-\infty}{\nabla}}_{-\infty}^{\alpha} \stackrel{t}{\nabla}^{\beta} f(t)=\stackrel{-\infty}{\nabla}^{\alpha+\beta} f(t)
$$

Proof. (i) is obvious.

(ii) Let $n_{1}, n_{2}$ be integers such that

$$
0<\operatorname{Re}\left(n_{1}-\alpha\right) \leq 1, \quad 0<\operatorname{Re}\left[n_{2}-(\alpha+\beta)\right] \leq 1,
$$

and let $n=\max \left\{0, n_{1}, n_{2}\right\}$.

First we note that the existence of

$$
\nabla_{-\infty}^{t} \stackrel{t}{\nabla}_{-\infty}^{\beta} f(t)
$$


is implied by assumptions (a) and (b). Then, by the definition,

$$
\begin{aligned}
& \underset{-\infty}{\stackrel{t}{\nabla}} \stackrel{\stackrel{t}{\nabla}}{\nabla_{-\infty}^{\beta}} f(t)=\lim _{a \rightarrow-\infty} \stackrel{t}{\nabla}_{a}^{\alpha} \lim _{b \rightarrow-\infty} \stackrel{t}{\nabla}_{b}^{\beta} f(t) \\
& =\lim _{a \rightarrow-\infty} \frac{\nabla^{n}}{\Gamma(n-\alpha)} \sum_{k=a}^{t}(t-k+1)_{n-\alpha-1} \\
& \quad \times \lim _{b \rightarrow-\infty} \frac{1}{\Gamma(-\beta)} \sum_{j=b}^{k}(k-j+1)_{-\beta-1} f(j) \\
& =\lim _{a \rightarrow-\infty} \frac{\nabla^{n}}{\Gamma(n-\alpha)} \\
& \quad \times \lim _{b \rightarrow-\infty} \frac{1}{\Gamma(-\beta)} \sum_{k=a}^{t}(t-k+1)_{n-\alpha-1} \sum_{j=b}^{k}(k-j+1)_{-\beta-1} f(j) .
\end{aligned}
$$

By the assumptions (a) and (b) we can let $a=b$. Then,

$$
\underset{-\infty}{\nabla^{\alpha}} \stackrel{t}{\nabla}_{-\infty}^{\beta} f(t)=\frac{\nabla^{n}}{\Gamma(n-\alpha)} \frac{1}{\Gamma(-\beta)} \lim _{a \rightarrow-\infty} \sum_{k=a}^{t} \sum_{j=a}^{k}(t-k+1)_{n-\alpha-1}(k-j+1)_{-\beta-1} f(j) .
$$

By the same argument as in the proof of Property 2(ii),

$$
\begin{aligned}
\underset{-\infty}{\nabla^{\alpha}} \underset{-\infty}{\nabla^{\beta}} f(t) & =\frac{\nabla^{n}}{\Gamma(n-\alpha-\beta)} \lim _{a \rightarrow-\infty} \sum_{j=a}^{t}(t-j+1)_{n-\alpha-\beta-1} f(j) \\
& =\frac{\nabla^{n}}{\Gamma(n-\alpha-\beta)} \lim _{a \rightarrow-\infty} \sum_{k=0}^{t-a}(k+1)_{n-\alpha-\beta-1} f(t-k)
\end{aligned}
$$

But $\lim _{a \rightarrow-\infty} \sum_{k=0}^{t-a}(k+1)_{n-\alpha-\beta-1} f(t-k)$ is convergent for any $t$ by assumption (c). It is then easy to show that

$$
\nabla^{n} \lim _{a \rightarrow-\infty} \sum_{k=0}^{t-a}(k+1)_{n-\alpha-\beta-1} f(t-k)=\lim _{a \rightarrow-\infty} \nabla^{n} \sum_{k=0}^{t-a}(k+1)_{n-\alpha-\beta-1} f(t-k) .
$$

Therefore,

$$
\begin{aligned}
\underset{-\infty}{t}{ }_{-\infty}^{t} \nabla^{\beta} f(t) & =\lim _{a \rightarrow-\infty} \frac{\nabla^{n}}{\Gamma(n-\alpha-\beta)} \sum_{k=0}^{t-a}(k+1)_{n-\alpha-\beta-1} f(t-k) \\
& =\nabla_{-\infty}^{t}{ }^{\alpha+\beta} f(t) .
\end{aligned}
$$

(iii) We have

$$
\begin{aligned}
& \stackrel{-\infty}{t}_{-\infty}^{\alpha} \nabla^{\beta} f(t)=\lim _{a \rightarrow-\infty} \stackrel{t}{a}^{\alpha} \nabla^{\beta} f(t) \\
& =\lim _{a \rightarrow-\infty} \frac{1}{\Gamma(-\alpha)} \sum_{k=a}^{t}(t-k+1)_{-\alpha-1} \sum_{l=0}^{\beta}(-1)^{l}\left(\begin{array}{l}
\beta \\
l
\end{array}\right) f(k-l) .
\end{aligned}
$$


By the same argument as in the proof of Property 2(iii),

$$
\begin{aligned}
& \nabla_{-\infty}^{t} \nabla^{\beta} f(t)=\lim _{a \rightarrow-\infty} \frac{1}{\Gamma(-\alpha)} \sum_{l=0}^{\beta}(-1)^{l}\left(\begin{array}{c}
\beta \\
l
\end{array}\right) \sum_{j=0}^{t-a}(j+1)_{-\alpha-1} f(t-l-j) \\
&=\lim _{a \rightarrow-\infty} \frac{1}{\Gamma(-\alpha)} \sum_{l=0}^{\beta}(-1)^{l}\left(\begin{array}{c}
\beta \\
l
\end{array}\right) {\left[\sum_{j=0}^{t-l-a}(j+1)_{-\alpha-1} f(t-l-j)\right.} \\
&\left.+\sum_{j=t-l-a+1}^{t-a}(j+1)_{-\alpha-1} f(t-l-j)\right] .
\end{aligned}
$$

Since

$$
\stackrel{-\infty}{\nabla-l}^{\alpha} f(t)=\lim _{a \rightarrow-\infty} \sum_{j=0}^{t-l-a} \frac{1}{\Gamma(-\alpha)}(j+1)_{-\alpha-1} f(t-l-j)
$$

exists, for any fixed $t$ and $l,(j+1)_{-\alpha-1} f(t-l-j)$ approaches zero as $j \rightarrow \infty$. Therefore, $\sum_{j=t-l-a+1}^{t-a}(j+1)_{-\alpha-1} f(t-l-j)$ approaches zero when $a$ goes to negative infinity. Thus the second limit in the above is zero. Hence,

$$
\begin{aligned}
\stackrel{-}{-\infty}^{\alpha} \nabla^{\beta} f(t) & =\lim _{a \rightarrow-\infty} \frac{1}{\Gamma(-\alpha)} \sum_{l=0}^{\beta}(-1)^{l}\left(\begin{array}{c}
\beta \\
l
\end{array}\right) \sum_{j=0}^{t-l-a}(j+1)_{-\alpha-1} f(t-l-j) \\
& =\lim _{a \rightarrow-\infty} \frac{\nabla^{\beta}}{\Gamma(-\alpha)} \sum_{j=0}^{t-a}(j+1)_{-\alpha-1} f(t-j) \\
& =\lim _{a \rightarrow-\infty} \stackrel{\nabla}{a}^{t+\beta} f(t) .
\end{aligned}
$$

Therefore,

$$
\underset{-\infty}{t} \nabla^{\alpha} \nabla^{\beta} f(t)=\stackrel{-\infty}{\nabla}_{-\infty}^{\alpha+\beta} f(t)
$$

A special case of Property $2^{\prime}$ is the following result.

PROPERTY $3^{\prime}$. If the assumptions of Property $2^{\prime}$ are satisfied for $\alpha$ and $f(t)$, then

$$
\stackrel{t}{\nabla}_{-\infty}^{t} \stackrel{t}{\nabla}_{-\alpha}^{-\alpha} f(t)=f(t)
$$

PROPERTY $4^{\prime}$. For $c$ a constant, if

$$
\stackrel{t}{\nabla^{\alpha}} f(t) \quad \text { and } \quad \stackrel{t}{\nabla} \alpha g(t)
$$

exist, then

$$
\underset{-\infty}{\stackrel{t}{\nabla}}[c f(t)+g(t)]=c \underset{-\infty}{t} \stackrel{-\infty}{\alpha}^{\alpha}(t)+\underset{-\infty}{t} \stackrel{-}{\alpha}^{\alpha}(t) .
$$

The proof is trivial.

PROPERTY $5^{\prime}$. If $\alpha$ is not a nonnegative integer and

$$
\lim _{\substack{a \rightarrow-\infty \\
b \rightarrow-\infty}} \sum_{n=0}^{t-a}\left(\begin{array}{l}
\alpha \\
n
\end{array}\right)\left[\stackrel{t-n}{\nabla}_{b}^{\alpha-n} f(t-n)\right]\left[\nabla^{n} g(t)\right] \quad \text { and } \lim _{b \rightarrow-\infty} \stackrel{t}{b}^{\alpha-n} f(t)
$$


exist for any fixed $t$, then

$$
\underset{-\infty}{t} \alpha(t) g(t)=\sum_{n=0}^{\infty}\left(\begin{array}{l}
\alpha \\
n
\end{array}\right)\left[\begin{array}{l}
t-n \\
\nabla_{-\infty}^{\alpha-n} f(t-n)
\end{array}\right]\left[\nabla^{n} g(t)\right]
$$

Proof. For any fixed $t$,

$$
\begin{aligned}
& \sum_{n=0}^{\infty}\left(\begin{array}{l}
\alpha \\
n
\end{array}\right)\left[\begin{array}{l}
t-n \\
\nabla_{-\infty}^{\alpha-n} f(t-n)
\end{array}\left[\nabla^{n} g(t)\right]\right. \\
& =\lim _{a \rightarrow-\infty} \sum_{n=0}^{t-a}\left(\begin{array}{l}
\alpha \\
n
\end{array}\right)\left[\lim _{b \rightarrow-\infty} \stackrel{\nabla}{\nabla}^{t-n} \alpha-n f(t-n)\right]\left[\nabla^{n} g(t)\right] .
\end{aligned}
$$

By the assumptions, we can let $a=b$. Thus the above expression equals

$$
\lim _{a \rightarrow-\infty} \sum_{n=0}^{t-a}\left(\begin{array}{l}
\alpha \\
n
\end{array}\right)\left[{\stackrel{\nabla}{\nabla^{-n}}}^{\alpha-n} f(t-n)\right]\left[\nabla^{n} g(t)\right] .
$$

By Property 5,

$$
\begin{aligned}
\sum_{n=0}^{\infty}\left(\begin{array}{l}
\alpha \\
n
\end{array}\right)\left[{ }_{-\infty}^{t-n}{ }^{\alpha-n} f(t-n)\right]\left[\nabla^{n} g(t)\right] \\
=\lim _{a \rightarrow-\infty} \stackrel{\nabla}{a}^{\alpha} f(t) g(t) \\
=\underset{-\infty}{\stackrel{t}{\nabla^{\alpha}} f(t) g(t) . \quad}
\end{aligned}
$$

Acknowledgements. The authors wish to thank Dr. Don McIntire for his helpful suggestions. Also the authors wish to acknowledge the referee for his comments which definitely improved the paper.

Department of Statistical Sciences

Southern Methodist University

Dallas, Texas 75275

1. J. B. Diaz \& T. J. Osler, "Differences of fractional order," Math. Comp., v. 28, 1974, pp. $185-201$.

2. C. W. J. GRANGER \& R. JOYEUX, "An introduction to long-memory time series models and fractional differencing," J. Time Ser. Anal., v. 1, 1980, pp. 15-29.

3. J. R. M. Hosking, "Fractional differencing," Biometrika, v. 68, 1981, pp. 165-176.

4. G. L. IsAACS, "Exponential laws for fractional differences," Math. Comp., v. 35, 1980, pp. 933-936. 